\title{
Racial Differences in Eligibility and Enrollment in a Smoking Cessation Clinical Trial
}

\author{
Andrea C. King, Dingcai Cao, \\ and Catherine C. Southard \\ University of Chicago
}

\author{
Alicia Matthews \\ University of Illinois at Chicago
}

\begin{abstract}
Objective: The purpose of this study was to compare the recruitment, eligibility screening, and enrollment of African American and White smokers into an intensive smoking cessation intervention trial [The Chicago STOP Smoking Trial (C-STOP)]. Methods: We compared demographic, smoking, substance use, and medical/psychiatric screening data from the recruitment records of 1,189 non-Hispanic, African American and White smokers screened for eligibility in the last year of a randomized pharmacological and behavioral smoking cessation trial. The study took place at a large urban medical center and two satellite locations within the Chicago metropolitan area. Results: Interest levels in the study were high among African American smokers, with twice as many African Americans as Whites contacting study staff for information and an initial screening. However, African Americans were nearly three times as likely not to be enrolled in the trial as Whites, because of higher ineligibility rates, failure to attend a screening session, and lower participation rates even among those meeting eligibility requirements. Conclusions: Racial differences were observed nearly at all levels of enrollment determination. These critical barriers to inclusion of African Americans in smoking cessation research limit our understanding of treatment efficacy and ultimately the ability to reduce the health disparities in tobacco-related disease experienced by African Americans.
\end{abstract}

Keywords: racial differences, smoking cessation, African American, eligibility, clinical trial

Cigarette smoking is recognized as the single most preventable cause of premature death in the United States (Centers for Disease Control and Prevention [CDC], 2005). Epidemiological studies suggest that the health burden associated with tobacco use is not evenly distributed across the entire population of smokers (CDC, 2004; CDC, 2005). In particular, African Americans experience more health burden from tobacco-related diseases compared with Whites and members of other ethnic minority groups (CDC, 2004; CDC, 2005). Lung Cancer rates among African Americans are significantly higher than Whites (Horner et al., 2009). African Americans also have a higher than expected prevalence of other

Accepted under the editorial term of Robert M. Kaplan.

Andrea C. King and Catherine C. Southard, Department of Psychiatry of Behavioral Neuroscience, University of Chicago; Dingcai Cao, Department of Surgery, Sections of Surgical Research and Ophthalmology \& Visual Science, University of Chicago; Alicia Matthews, College of Nursing, University of Illinois at Chicago.

This study was supported by a grant from the National Institute of Drug Abuse (\#R01-DA016834). We thank the Howard Brown Health Center and Respiratory Health Association of Metropolitan Chicago for their overall support and for providing satellite study locations. Appreciation is extended to our colleague Dr. Susan Everson-Rose for her critical comments on the manuscript. Finally, we thank Sarah Butler for technical and administrative assistance and to Ryan Stachoviak, Ayodele Gomih, Michelle Carpentier, Toni Harris, and Constantine Trela for their role in data collection and database management.

Correspondence concerning this article should be addressed to Andrea C. King, Department of Psychiatry and Behavioral Neuroscience, University of Chicago, 5841 S. Maryland Avenue (MC-3077), Chicago, IL 60637. E-mail: aking@bsd.uchicago.edu health problems, such as obesity, which may increase risk and mortality from diseases associated with or exacerbated by smoking (e.g., heart disease, diabetes, asthma, certain cancers and HIV/ AIDS; CDC, 2005; Targonski, Persky, Orris, \& Addington, 2005). In addition to these more severe health outcomes, the prevalence of tobacco smoking among African Americans has not declined to extent of Whites over the last few decades (CDC, 2005). Factors associated with poverty (e.g., reduced access to treatment) may play a role in the widening disparities in tobacco cessation across population groups. Smoking rates are significantly higher for persons living below the poverty line than for those living above the poverty line (30\% vs. 22\%; CDC, 2007). In the United States, while a greater percentage of Whites versus African Americans under the poverty line are current smokers (42\% vs. $33 \%$; Barbeau, Krieger, \& Soobader, 2004), there are disproportionately more African Americans than Whites who live in poverty (33\% vs. $12 \%$, respectively; Kaiser Family Foundation, 2008). African Americans from lower socioeconomic groups also have some of the highest levels of adverse smoking outcomes in the United States (CDC, 2007). Statistically controlling for socioeconomic factors has been shown to reduce, but not completely ameliorate, the racial differences in smoking quit rates (King, Polednak, Bendel, Vilsaint, \& Nahata, 2004); however, this has not been found in all studies (Kiefe et al., 2001).

Despite disproportionate levels of tobacco usage and more severe health burdens from tobacco-related disease (Fiore et al., 2008), adequate representation of African Americans in most pharmacological smoking cessation clinical trials has been lacking. For example, large-scale trials establishing efficacy of the Food and Drug Administration (FDA)-approved medications bupropion 
(Hurt et al., 1997; Jorenby et al., 1999) and varenicline (Gonzales et al., 2006; Jorenby et al., 2006; Tonstad et al., 2006) for treating tobacco dependence have included only $2 \%$ to $11 \%$ African Americans in their samples. Similarly, nicotine replacement therapy studies have not included sufficient numbers of African Americans and non-Whites (3.3\%-10.3\%) (Shiffman et al., 2002a; Shiffman et al., 2002b) or failed to report on race distributions within their samples (Hjalmarson, Franzon, Westin, \& Wiklund, 1994; Schneider et al., 1996; Tonnesen, Norregaard, Mikkelsen, Jorgensen, \& Nilsson, 1993). In studies examining the potential role of the opioid antagonist naltrexone for smoking cessation, two studies did not report on racial composition (Covey, Glassman, \& Stetner, 1999; Wong et al., 1999) and two other studies ranged from $6.5 \%$ (O'Malley et al., 2006) to $27.2 \%$ (King et al., 2006) African Americans. Thus far, the source of the lack of adequate representation of African Americans in smoking cessation trials is unclear, as to our knowledge, systematic study of the effects of race at each step in the enrollment process has not been undertaken among smoking cessation clinical trials.

Minority enrollment in research, particularly for African Americans, has increased in the 15 years since National Institutes of Health (NIH) policies required their inclusion (Dickerson, Leeman, Mazure, \& O'Malley, 2009; Nollen et al., 2006). Moreover, several studies have been successful in exclusively enrolling African American smokers and have demonstrated efficacy of nicotine replacement and bupropion, albeit at lower quit rates compared with mainly White samples (Ahluwalia, Harris, Catley, Okuyemi, \& Mayo, 2002; Ahluwalia, McNagny, \& Clark, 1998). Ideally, clinical trials should include adequate representation of both White and non-White smokers to allow direct comparisons of eligibility, enrollment, follow-up rates, and treatment outcomes (Benowitz, 2002). However, the majority of studies are hindered by low representation of non-Whites, because of difficulty in minority recruitment and retention, low program completion and follow-up rates, and lower acceptance of and adherence to pharmacological treatments (Ahluwalia et al., 2002; Robles, SinghFranco, \& Ghin, 2008; Shaya, Gbaravor, Huiwen, Agyeman-Dauh, $\&$ Saunders, 2007). There is a critical need to improve recruitment and retention of African American smokers in treatment trials in order for conclusions to be drawn regarding efficacy of particular treatments for this underserved subgroup and to reduce their health disparities in tobacco-related disease and early mortality. Gaps in the extant literature include identifying effective outreach and recruitment strategies for African American smokers, understanding the temporal barriers during eligibility determination, and discerning the influence of racial versus within-group demographic characteristics associated with enrollment.

The purpose of this study was to compare the recruitment, screening, and enrollment of African American and White smokers into an intensive smoking cessation intervention trial [The Chicago STOP Smoking Trial (C-STOP); (King \& Cao, 2010)]. Subjects were study candidates who contacted research staff to obtain information and participate in an initial telephone screening interview to ascertain eligibility in the trial during the last year of enrollment. Specific questions in this study addressed whether, above and beyond sociodemographic differences between African Americans and Whites, there were racial differences in: (a) the prevalence of telephone screening candidates and outreach sources that generated study awareness, (b) interest in quitting smoking and other background characteristics, (c) attendance and eligibility rates, and (d) reasons for ineligibility. This information has important implications for future research aimed at increasing access to and participation in clinical trial research for African American smokers.

\section{Method}

\section{Design and Procedures}

This study was part of C-STOP, a double-blind, randomized placebo-controlled trial of the efficacy of the oral opioid antagonist, naltrexone, in combination with nicotine patch and individual behavioral counseling (King \& Cao, 2010). The sample included in this article consisted of telephone screening candidates during the last year of C-STOP enrollment, from March 1, 2008, to February 28, 2009. Because C-STOP enrolled participants regardless of race or ethnicity, during the first $11 / 2$ years of the trial, these background questions were not included in the telephone screening but were later added to systematically compare recruitment and enrollment in African American and White smokers. In the overall randomized clinical sample of 315 smokers, 181 were White (57\%), 110 (35\%) were African American, and 24 (8\%) were of other racial/ethnic background. African American enrollment was steady throughout the trial and did not differ between the initial and later recruitment intervals (36\% vs. $33 \% ; \chi^{2}=0.75$, $p=.39)$. It is important to note that the proportion of African American smokers randomized in C-STOP was 2 to 3 times higher than in most prior pharmacological and behavioral smoking cessation trials (Gonzales et al., 2006; Hurt et al., 1997; Jorenby et al., 2006; Jorenby et al., 1999; O’Malley et al., 2006; Tonstad et al., 2006).

\section{Recruitment}

Advertisements included information that the study was enrolling generally healthy adult smokers who desired to quit, were 18-65 years old, and smoked 12 to 40 cigarettes daily for 2 or more years. Per institutional review board (IRB) requirements, the study drug (naltrexone) was not named on any recruitment materials because it is not currently FDA-approved for smoking cessation. The study included three sites, the primary site at the University of Chicago Clinical Addictions Research Laboratory, located on the South side of Chicago, and two secondary sites at nonprofit organizations-the Respiratory Health Association of Metropolitan Chicago in downtown Chicago and the Howard Brown Health Center on the North side of Chicago. All sites were accessible by public transportation and/or had parking facilities within proximity. Enrollment of African Americans in C-STOP was higher at the primary site $(53 \%$; $99 / 188)$ than the two secondary sites $[25 \%(19 / 75)$ at the downtown site and $2 \%(1 / 46)$ at the North side site], consistent with neighborhood demographics in these metropolitan area regions (U.S. Census Bureau, 2000).

Efforts were made to recruit a racially diverse sample of smokers. Based on successful recruitment of minority smokers in our prior studies (King et al., 2006; King, Sánchez-Johnsen, Van Orman, Cao, \& Matthews, 2008) and the critical need for greater enrollment of African Americans in smoking cessation trials (Dickerson et al., 2009), our goal was to enroll approximately 
one-third African Americans into the trial. Best practice strategies employed by the research team included using general as well as culturally relevant recruitment materials (Matthews, SánchezJohnsen, \& King, 2009) based on the work of Kreuter and colleagues (Kreuter, Lukwago, Bucholtz, Clark, \& SandersThompson, 2003), hiring a recruitment specialist, and having a diverse team of research assistants (i.e., 2 African American women, 1 White woman, and 2 White men). Post hoc analyses revealed no differences in subject eligibility determination rates based on the race or gender of the research assistant conducting the assessments and interviews.

Three general types of recruitment approaches for recruiting all subjects, regardless of race/ethnicity were used: clinic-based recruitment and active and passive community outreach methods (Harris et al., 2003; Lee et al., 1997; Webb, Seigers, \& Wood, 2009). To increase enrollment of underserved minority smokers, communities on the South side of Chicago with high concentrations of low-to-middle income African American smokers were specifically targeted (U.S. Census Bureau, 2000). Recruitment sources were classified into seven categories: physician or health care professional referral, community health fairs or local flyers, public transportation advertisement, traditional media advertisement (newspaper, TV or radio), digital media advertisement (Internet sites), word of mouth, and other miscellaneous sources.

\section{Telephone Screening}

Similar to most trials, enrollment of participants took place after eligibility for inclusion was determined in two phases: telephone screening followed by in-person screening. During the telephone interview, interested candidates were provided information on the purpose of the study, the study drug (naltrexone or placebo) and general requirements for participation. They also were informed that participation required willingness to attend 10 study visits and 2 follow-ups, to complete study measures, and be randomized to study medication or placebo. After this initial information exchange, under the auspices of a waiver of consent authorization from the University of Chicago IRB, the trained research assistant conducted a 10-min confidential telephone interview with the candidate to ascertain eligibility based on the candidate's demographics, smoking history, desire to quit, general health status including psychiatric, medical, and substance use history, and current medications. Telephone screening eligibility included: ages 18 to 65 inclusive; smoke at least 12 but no more than 40 cigarettes daily for at least two years; no major medical or psychiatric conditions or medications, opioid dependence, or chronic opioid use; willingness to participate and a stable residence and/or telephone number (self or friend). For data coding purposes, ineligibility reasons were categorized into four distinct categories, including smoking patterns, substance use, medical/psychiatric factors, and other rejection reasons (scheduling difficulties, loss of interest, and related miscellaneous factors). Ineligible study candidates were thanked for their time and given information on local stop smoking programs, but to maintain integrity of screening procedures they were not told specifically why they did not qualify.

For approximately $85 \%$ of eligible candidates, the subsequent in-person screening and orientation session was scheduled at the conclusion of the telephone screen. For those unable to schedule an in-person screening, up to three additional contacts were attempted to schedule the visit. Candidates were told they would receive travel compensation but not be paid for the in-person screening. Those who cancelled their screening visit had up to two more opportunities to reschedule before they were classified as no longer interested.

\section{In-Person Screening and Orientation}

The in-person screening took place at the primary study site as it included appropriate facilities and staffing for the medical portion of the screening. After the research assistant described the study details, the candidate signed the consent form and then participated in questionnaires and interviews for psychiatric and medical status, a brief physical examination, a urine toxicology screen and pregnancy test, a blood sample for blood chemistries and hepatic profile, and an expired-air carbon monoxide test $(\mathrm{CO}$; Smokerlyzer, Bedfont Scientific, Medford NJ). Candidates also had height and weight measured, and BMI was calculated (Garrow \& Webster, 1985).

A trained diagnostic assessor administered the modified version of the structured clinical interview for Structured Clinical Interview for the Diagnostic and Statistical Manual of Mental Disorders, Fourth Edition (DSM-IV; SCID) (First, Spitzer, Gibbon, \& Williams, 1995). Candidates were deemed ineligible if they did not meet the aforementioned inclusion criteria, as well as if they met criteria for past year major Axis I disorder or had a history of severe Axis II disorder, had a body mass index (BMI) outside the range of 18 to 38 , had an expired air $\mathrm{CO}$ reading of $<10 \mathrm{ppm}$, or had a positive urine toxicology screen (except for marijuana). Marijuana use was allowed as long as the frequency was not more than 4 times weekly, and the candidate did not meet criteria for cannabinoid dependence. Ineligible candidates were thanked for their time, compensated for travel, and offered other stop smoking referrals, as warranted.

\section{Study Enrollment}

Candidates interested in enrolling and determined appropriate for inclusion were eligible to participate in the C-STOP clinical trial. The participant chose which of the three study sites they preferred for their visits. The trial included six individual behavioral counseling sessions, study drug for 13 weeks, and nicotine patch (starting at $21 \mathrm{mg}$ and tapering down to $7 \mathrm{mg}$ at four weeks). Subjects were compensated for travel expenses and received $\$ 110$ for completing study measures during treatment, and an additional $\$ 60$ for attending each of the 6- and 12-month follow-ups.

\section{Statistical Analyses}

Background characteristics were compared between African American and White study candidates $(N=1,189)$ using Student $t$ tests and/or $\chi^{2}$ tests, where appropriate. Logistic regression models were used to compare African Americans and Whites on ineligibility, nonattendance, and nonenrollment rates. Multinomial logistic regression models were used to compare groups on polytomous responses, including referral sources and reasons for ineligibility. For each outcome, univariate analyses were initially conducted, and then followed by multivariate analyses examining 
the effect of race on the outcome while controlling for other significant sociodemographic factors that differed between the races or related to the outcome. The main sociodemographic factors examined were race, age, gender, estimated household income, cigarettes per day and desire to quit. For logistic regression, odds ratios (OR) between African Americans and Whites were reported and for multinomial logistic regression, relative risk ratios (RRR) were reported.

\section{Results}

\section{Recruitment}

Participants in this study were 1,189 non-Hispanic telephone screen candidates who identified their race as either Black/African American $(n=819,62 \%)$ or White/Caucasian $(n=370,38 \%)$. In terms of referral sources, $49 \%$ of the sample cited public transportation advertisement as the main source for their awareness of the study, followed by traditional media advertisement (17\%) and word of mouth referrals $(15 \%)$ as the next most frequently cited sources. All other referral sources were cited in $<6 \%$ of telephone candidates. Referral source was significantly associated with race, as well as gender, age, education, income, and cigarettes per day. After controlling for these latter variables, race remained significantly associated with referral method. In particular, compared with Whites, African Americans were more likely to: (a) use traditional media, public transportation advertisement, or word-ofmouth referral than digital media advertisement; traditional media: $\mathrm{RRR}=3.35$ (95\% confidence interval $[\mathrm{CI}]=1.53-7.34), p<.01$; public transportation: $\mathrm{RRR}=3.61(95 \% \mathrm{CI}=1.75-7.47), p=$ .001 ; word-of-mouth: $\mathrm{RRR}=2.92(95 \% \mathrm{CI}=1.33-6.42), p<$ .01 ; and (b) use public transportation advertisement than physician referral, $\mathrm{RRR}=2.19(95 \% \mathrm{CI}=1.14-4.20), p<.05$.

\section{Enrollment Stages and Overall Participation Rate}

Figure 1 depicts a Consolidated Standards of Reporting Trials diagram including eligibility for African Americans and Whites in C-STOP at each phase in the determination and enrollment process. Overall, African Americans were over three times less likely to enroll in C-STOP than Whites (4\% versus $17 \%$, respectively). Other factors associated with nonenrollment included fewer years
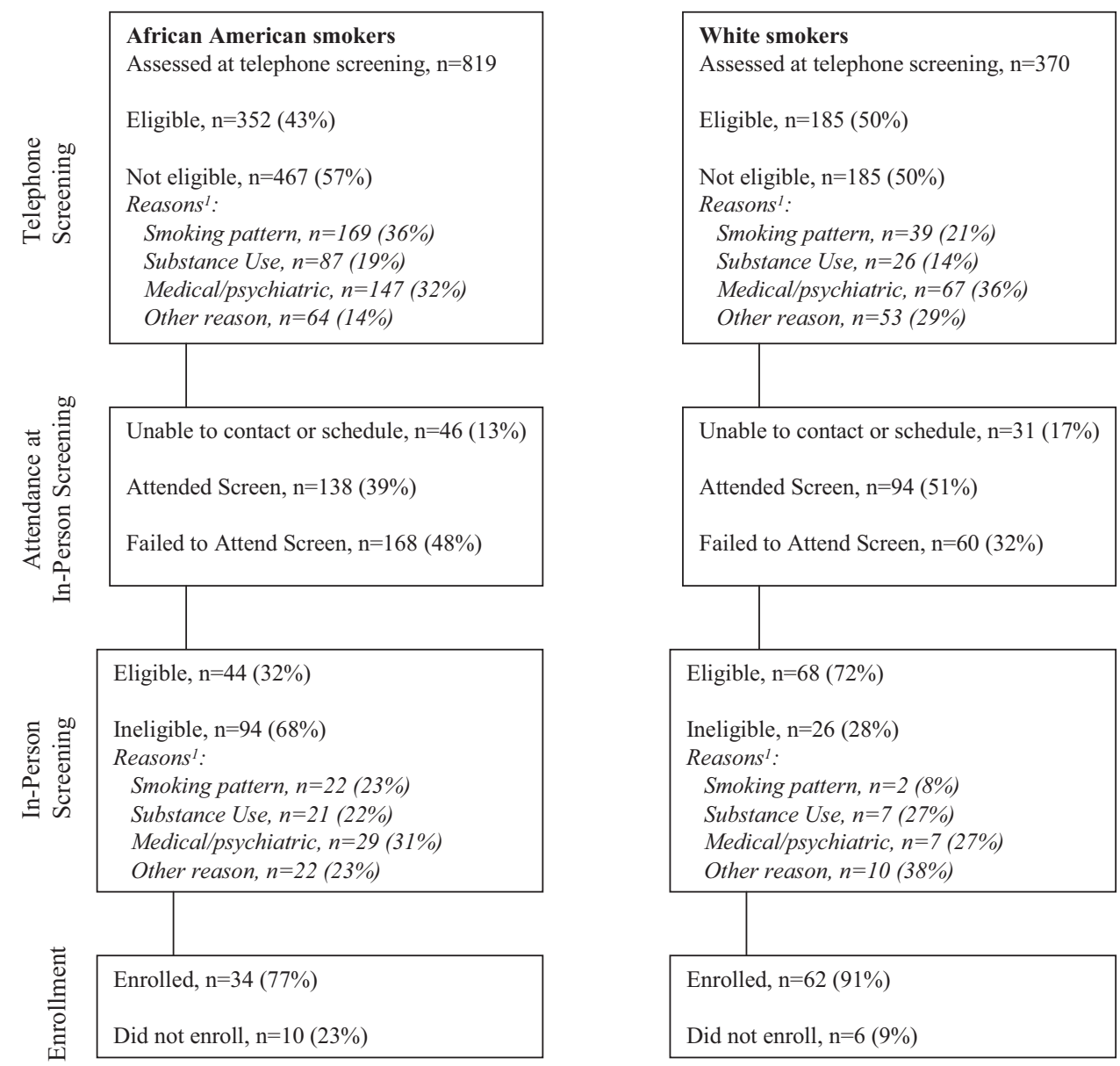

Figure 1. Consolidated Standards of Reporting Trials diagram of eligibility determination in 1,189 nonHispanic African American and White smokers. Stages include telephone screening eligibility, attending the in-person screening, in-person screening eligibility, and electing to enroll in the trial among those deemed eligible. ${ }^{1}$ Due to rounding, $\%$ totals may not equal $100 \%$ in all cases. 
of education, lower estimated household income, and fewer cigarettes smoked per day. Even after controlling for these factors, African Americans were nearly three times as likely to not enroll in the trial compared with Whites (Table 1). To examine the potential source of the race difference in overall enrollment, we examined the groups on sample characteristics and eligibility rates at telephone screening, attendance and eligibility rates at in-person screening, and enrollment among those meeting all eligibility criteria.

\section{Sample Characteristics of Those Receiving Initial Telephone Screening Interview}

Relative to Whites, African American smokers included fewer men $(57.8 \%$ vs. $66.7 \%, p=.004)$, and were older $(43.7 \pm 10.5$ vs. $37.0 \pm 12.6$ years, $p<.001)$, less educated $(13.0 \pm 1.8$ vs. $14.6 \pm$ 2.3 years, $p<.001$ ), and smoked fewer cigarettes per day (16.4 \pm 8.3 vs. $18.9 \pm 7.6, p<.001)$. African Americans endorsed higher levels of interest in quitting, with $80.1 \%$ self-reporting a high desire to quit smoking (rating of 10 on an ascending 10-point scale) compared with $62.3 \%$ of Whites, $\chi^{2}(1)=41.27, p<.001$. Based on 2000 U.S. Census data on household income averages for each candidate's reported zip code for primary residence (U.S. Census Bureau, 2000), African Americans had a lower estimated median household income than Whites $(\$ 33.1 \mathrm{~K} \pm 9.6 \mathrm{~K}$ vs. $\$ 42.5 \mathrm{~K} \pm 11.4 \mathrm{~K} ; p<.001)$.

\section{Eligibility Determination at Initial Telephone Screening Interview}

During the telephone screening, 57\% African Americans and $50 \%$ Whites did not meet study eligibility criteria. Ineligibility at the initial screening was significantly associated with African American race, older age, fewer years of education, male gender, lower income, and fewer cigarettes smoked per day (Table 1). After controlling for the latter five sociodemographic variables, African Americans were in fact more likely than Whites to be determined eligible during the initial telephone screening interview, indicating that the lower rate of meeting criteria at this stage for African Americans was not because of racial bias but rather a product of the sociodemographic differences between them and Whites. The distributions of reasons for ineligibility did differ significantly between the races, $\chi^{2}(3)=28.48, p<.001$, as well as by age, gender, income and smoking level. Compared with Whites, African Americans were more likely to be rejected because of both lighter smoking patterns, RRR $=2.01(95 \% \mathrm{CI}=$ 1.06-3.77), $p=.031$, and substance use factors, $\mathrm{RRR}=3.16$ (95\% CI $=1.59-6.28), p=.001$, relative to other rejection reasons (Figure 1).

\section{Attendance at the In-Person Screening Visit}

African Americans and Whites did not differ on the percentage of telephone candidates who were unable to be scheduled for the in-person screening visit, $\mathrm{OR}=0.75$ (95\% CI: $0.44-1.27$ ), $p=$ .246. However, among those scheduled, African Americans were significantly more likely to fail to attend than Whites (Table 1). Screening visit nonattendance was also associated with younger age and fewer years of education. However, even after controlling

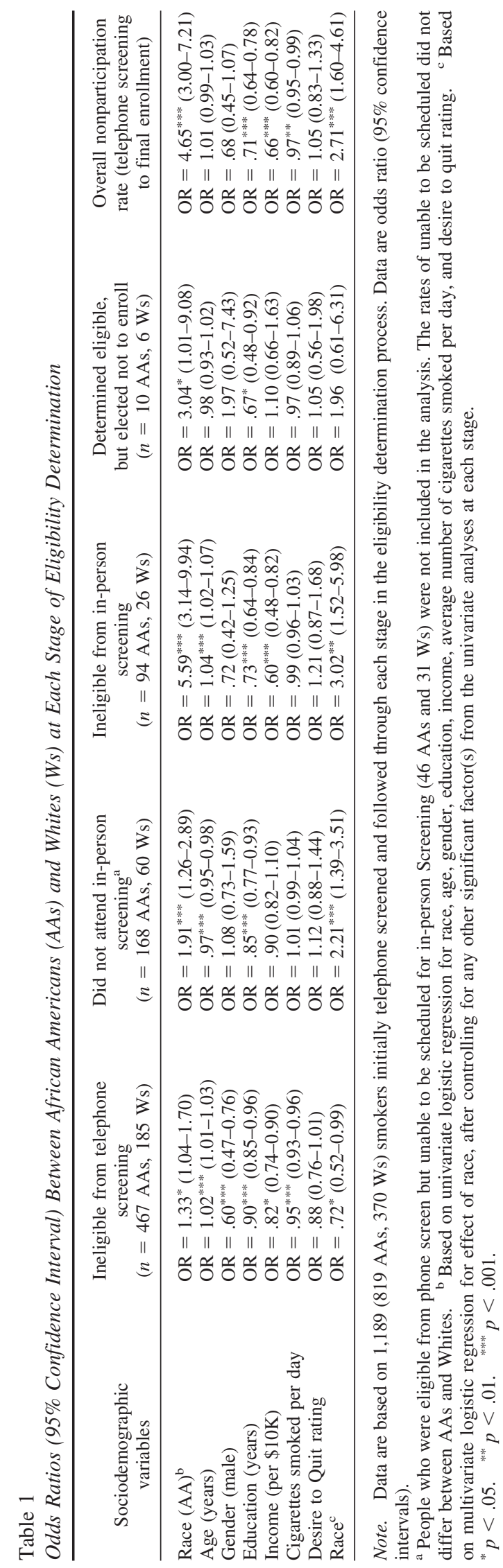


for these factors, nonattendance rates for screening remained higher in African Americans than Whites (48\% vs. 32\%, respectively).

\section{Eligibility Determination at In-Person Screening Visit}

Of the candidates who attended the in-person screen, African Americans were significantly more likely than Whites to be determined ineligible, $72 \%$ (94/138) versus $28 \%$ (26/94), respectively. However, the distribution of the reasons for ineligibility at in-person screening did not differ by race (Figure 1). In addition to race, ineligibility at the in-person screening also was associated with older age, fewer years of education, and lower income. After controlling for these factors, African Americans were still twice as likely to be determined ineligible at in-person screening compared with Whites.

\section{Study Eligibility but Nonenrollment}

The final set of analyses pertained to study candidates who successfully met eligibility criteria at the in-person screen but elected not to enroll in the trial. Both African American race and fewer years of education were associated with non enrollment in C-STOP among those determined eligible. Once education level was taken into account, race was no longer a significant predictor of study enrollment (Table 1).

\section{Discussion}

Historically, participation by African Americans and other racial/ethnic minorities in health-related Phase III clinical trials has been limited (Shaya et al., 2007). To our knowledge, this article provides the first systematic comparison of barriers to enrollment at multiple screening stages in African Americans and Whites within a large smoking cessation intervention trial. The present study adds to the extant literature with key findings of racial differences among cited referral source, demographic and smoking characteristics, and attendance and eligibility rates. While we were successful in enrolling a high proportion of African American smokers in C-STOP (35\%; King, Cao, Southard, \& Matthews, 2010), we nonetheless observed significant racial disparities at several stages in eligibility determination and in overall study enrollment rates. Even after controlling for several sociodemographic factors that differed between African American and White study candidates, such as education, income, and smoking level, African Americans remained nearly three times as likely to not enroll in C-STOP as Whites (Table 1). Given that a smaller proportion of African American candidates were enrolled versus Whites (4\% vs. 17\%, respectively), which is likely the case in other trials, the generalizability of findings and their implications may be reduced among African American smokers.

Lower enrollment of African Americans into the trial appeared to be because of two main factors: higher rates of ineligibility and poorer attendance at the in-person screening session. Although African Americans were less likely to be eligible at telephone screening than Whites, multivariate analyses controlling for age, sex, education, income and cigarettes smoked per day revealed that the eligibility disparity for African Americans was no longer evident at this initial telephone screening stage, in fact, after controlling for these sociodemographic factors, African Americans were more likely than Whites to be eligible and agree to schedule the next step in the determination process, that is, the in-person screening visit. This finding is similar to work by others indicating that differences between the races may be reduced after other sociodemographic factors are taken into account (King et al., 2004), which thereby reduces the likelihood that racial bias was evident during the initial contact or that biological or genetic effects may underlie effects. However, despite strong reported interest levels, African Americans who were eligible for the inperson screening were less likely to attend the appointment than Whites; a finding that was also reported in another independent study (Gariti et al., 2008).

The larger C-STOP intervention study was designed to evaluate the impact of a combined behavioral and pharmacological treatment on smoking quit rates in a diverse sample of adult smokers. As such, evaluation of sociocultural variables that may account for barriers to trial enrollment was beyond both the scope of the main trial as well as the auspices of a waiver of consent authorization from the IRB to inquire on the initial telephone interview only about pertinent general inclusion and exclusion criteria. However, the extant literature on barriers within minorities may help to inform our team's and others future research in this area. For example, based on prior research, we may speculate that the failure to attend this visit may be because of heightened barriers in African Americans versus Whites in terms of childcare costs (Woods et al., 2002), medical mistrust (Woods et al., 2002), perceived stress (Lacey et al., 1993), and less knowledge about negative health consequences of smoking and benefits of smoking cessation interventions (Fagan et al., 2004; Mazas \& Wetter, 2003). African Americans may exhibit less receptivity to formal smoking cessation interventions (Audrain-McGovern, Halbert, Rodriguez, Epstein, \& Tercyak, 2007; Dahm et al., 2009; Fu et al., 2008), and this could also have factored in their nonattendance at the screening visit. Furthermore, among those who did attend screening, African Americans fared worse than Whites in study enrollment: even after controlling for sociodemographic factors, they were twice as likely to be determined ineligible. While the reasons for in-person ineligibility did not differ statistically between the races, it appears to be mainly attributable to the fact that African Americans smoked too few cigarettes daily to meet eligibility (23\% vs. $8 \%$ in Whites). This issue, and suggestions for ameliorating it within future trials, is discussed in more detail later in this section.

Contrary to many previously reported trials, we experienced high levels of interest in smoking cessation trial participation among African American smokers, with twice as many African Americans as Whites contacting the study to complete an initial telephone screening. Best practices for recruiting diverse samples were used to foster knowledge about the study including the distribution of both generic and culturally targeted materials, engaging multiple outreach strategies, and employing a diverse research staff (Fitzgibbon et al., 1998; Harris et al., 2003; Jeffries, Choi, Butler, Harris, \& Ahluwalia, 2005; Staffileno \& Coke, 2006; Webb et al., 2009). African Americans were significantly more likely than Whites to cite awareness of the study through public transportation advertisement, traditional media, and word of mouth referrals than by digital media, which is consistent with prior 
research indicating a potential "digital divide" among racial/ethnic minorities versus Whites (Brodie et al., 2000; Lorence, Park, \& Fox, 2006). Furthermore, enrollment of African Americans and Whites across study sites were consistent with metropolitan area demographics, with a lower proportion of African Americans enrolling at the North side site compared with the primary site on the South side of Chicago (adjacent to a high concentration of predominantly African American neighborhoods; U.S. Census Bureau, 2000), with the downtown site intermediate. In sum, the findings highlight the importance of informal, traditional, and multifaceted approaches (i.e., radio, print) for increasing outreach into African American communities as well as consideration of multiple study sites, if possible, to ensure diversity.

Consistent with epidemiological and population statistics (CDC, 2009; U.S. Census Bureau, 2000), African American smoker candidates smoked fewer cigarettes per day, and had lower education levels and estimated household incomes than Whites. Smoking statistics in the United States show higher smoking rates are related to lower socioeconomic status and education levels, and in younger persons (CDC, 2009). However, African American candidates in this study were also significantly older than Whites. This finding is consistent with data suggesting a lower overall percentage of ever smokers that have quit smoking within African Americans compared with other racial/ethnic groups (King, Polednak, Bendel, Vilsaint, \& Nahata, 2004). Regarding smoking patterns, the eligibility criteria for C-STOP of between 12 and 40 daily cigarettes smoked was similar to most smoking cessation trials (Fiore et al., 2008). This range was used to minimize sample heterogeneity and ensure safety with nicotine replacement which was initiated at the highest dose $(21 \mathrm{mg} /$ day $)$. However, while African Americans may smoke fewer cigarettes than Whites, they often have similar or even greater nicotine dependence (Royce, Hymowitz, Corbett, Hartwell, \& Orlandi, 1993). Several factors may contribute to observed racial differences in nicotine dependence by race. For example, data suggests there are differences in nicotine metabolism between African American and Caucasian smokers (Dickerson, Leeman, Mazure, O’Malley, 2009; Piper, Fox, Welsch, Fiore, \& Baker, 2001; Signorello, Cai, Tarone, McLaughlin, \& Blot, 2009; Wagenkneckt et al., 1990). Specifically, African American smokers have been found to have cotinine levels equal to or greater than Caucasian smokers even though they report smoking at lower levels. Racial differences in smoking topography and metabolism has significance for the inclusion/ exclusion of African American smokers in clinical trials if the criteria for inclusion focus only on level of smoking. Additionally, differences in dependency levels may be influenced by the disproportionate use of menthol cigarettes by African Americans (Clark, Gautham, \& Gerson, 1996; Gandhi, Foulds, Steinberg, Lu, \& Williams, 2009). Menthol is known to have an anesthetic effect and increased puff volume absorption and hence high nicotine levels in the blood (Giovino et al., 2004). Further research is needed to improve assessment of nicotine dependency, administration of appropriate levels of nicotine replacement therapies and determination of eligibility classifications for African American smokers in smoking research trials.

The findings from this article contribute substantially to the extant literature by identifying racial differences between African American compared with White smokers during the multiple stages of recruitment, screening, eligibility determination, and eventual enrollment into a smoking cessation intervention trial. Strengths of this study included adequate representation of African American study candidates, high interest levels for the trial, and multiple stage analyses of racial differences. However, limitations included the brief nature of telephone screening, assessing general eligibility without being invasive of privacy, but not including complex sociodemographic variables of interest, such as level of acculturation, health care access, perceptions of discrimination, and trust in the medical system. This study was also limited by lack of inclusion of sufficient numbers of other minority groups, such as American Indian/Alaskan Native or Hispanics, who are underrepresented in smoking cessation research (Lacey et al., 1993) and also experience disproportionate tobacco-related health consequences. While there are no formal mandates to include such information, if more trials would report on the sociodemographic characteristics of the candidate pool compared with enrolled participants, the full scope of racial/ethnic differences may be better highlighted and lead to improved culturally appropriate strategies to reduce selection bias.

In summary, overall study findings may have important implications for increasing the participation of African Americans in clinical trials. Similar to previous studies that reported on the successful recruitment of African Americans to clinical trials (Ahluwalia et al., 1998; Audrain-McGovern et al., 2007; Dahm et al., 2009; Fagan et al., 2004; Mazas \& Wetter, 2003), we used a multifaceted recruitment approach that yielded a large and diverse sample of African Americans. Nevertheless, noneligibility and nonattendance rates were significantly heightened in African Americans. African American representation in future smoking trials may be enhanced by: (1) including a broader range of smokers to include lighter smokers, whose prevalence is increasing (Shiffman, 2009); (2) employing alternate strategies for smoking determination, such as cotinine (Pérez-Stable, Herrera, Jacob, \& Benowitz, 1998; Robles et al., 2008) levels or scores on nicotine dependence scales (Heatherton, Kozlowshi, Frecker, \& Fagerström, 1991); and (3) having greater flexibility in medication dosing to accommodate more diversity of smoking patterns. A broader inclusion approach will not only increase clinical relevance, but also reduce racial/ethnic disparities in enrollment to facilitate development of appropriate smoking cessation interventions (Fagan et al., 2004). The public health relevance of greater inclusion of African Americans and other minorities in smoking cessation trials may reduce racial disparities in outcomes, and ultimately to reduce tobacco-related disease burden among such underserved populations.

\section{References}

Ahluwalia, J. S., Harris, K. J., Catley, D., Okuyemi, K. S., \& Mayo, M. S. (2002). Sustained-release bupropion for smoking cessation in African Americans. Journal of the American Medical Association, 288, 468474.

Ahluwalia, J. S., McNagny, S. E., \& Clark, W. S. (1998). Smoking cessation among inner-city African Americans using the nicotine transdermal patch. Journal of General Internal Medicine, 13, 1-8.

Audrain-McGovern, J., Halbert, C., Rodriguez, D., Epstein, L. H., \& Tercyak, K. P. (2007). Predictors of participation in a smoking cessation program among young adult smokers. Cancer Epidemiology, Biomarkers, \& Prevention, 16, 617.

Barbeau, E. M., Krieger, N., \& Soobader, M. J. (2004). Working class 
matters: Socioeconomic disadvantage, race/ethnicity, gender and smoking in NHIS 2000. American Journal of Public Health, 94, 269-278.

Benowitz, N. L. (2002). Smoking cessation trials targeted to racial and economic minority groups. Journal of General Internal Medicine, 288, 497-499.

Brodie, M., Flournoy, R. E., Altman, D. E., Blendon, R. J., Benson, J. M., \& Rosenbaum, M. D. (2000). Health information, the Internet, and the digital divide. Health Affairs, 19, 255-265.

Centers for Disease Control and Prevention. (2009). Vital and Health Statistics, Series 10, Number 242, Summary Health Statistics for U.S. Adults: National Health Interview Survey, 2008. Publication No. (PHS) 2010-1570. Washington, DC: U.S. Department of Health and Human Services.

Centers for Disease Control and Prevention. (2004). State-specific prevalence of current cigarette smoking among adults-United States, 2002. Morbidity and Mortality Weekly Review, 52, 1277-1280.

Centers for Disease Control and Prevention. (2005). Annual smoking: Attributable mortality, years of potential life lost, and productivity losses-United States, 1997-2001. Morbidity and Mortality Weekly Review, 54, 625-628.

Centers for Disease Control and Prevention. (2007). Cigarette smoking among adults-United States, 2006. Morbidity and Mortality Weekly Review, 56, 1157-1161.

Clark, P. I., Gautham, S., \& Gerson, L. W. (1996). Effect of menthol cigarettes on biochemical markers of smoke exposure among black and white smokers. Chest, 110, 1194-1198.

Covey, L. S., Glassman, A. H., \& Stetner, F. (1999). Naltrexone effects on short-term and long-term smoking cessation. Journal of Addictive Diseases, 18, 31-40.

Dahm, J. L., Cook, E., Baugh, K., Wileyto, E. P., Pinto, A., Leone, F., \& Schnoll, R. A. (2009). Predictors of enrollment in a smoking cessation clinical trial after eligibility screening. Journal of the National Medical Association, 101, 451-455.

Dickerson, D. L., Leeman, R. F., Mazure, C. M., \& O’Malley, S. S. (2009). The inclusion of women and minorities in smoking cessation clinical trials: A systematic review. American Journal on Addictions, 18, 21-28.

Fagan, P., King, G., Lawrence, D., Patrucci, S. A., Robinson, R. G., Banks, D., \& Grana, R. (2004). Eliminating tobacco-related health disparities: Directions for future research. American Journal of Public Health, 94, 211-217.

Fiore, M. C., Jaén, C. R., Baker, T. B., Bailey, W. C., Benowitz, N. L., Curry, S. J., \& Wewers, M. E. (2008). Treating tobacco use and dependence: 2008 update. Clinical Practice Guideline. Rockville, MD: U.S. Department of Health and Human Services, Public Health Service.

First, M. B., Spitzer, R. L., Gibbon, M., \& Williams, J. B. W. (1995). Structured clinical interview for DSM-IV-TR Axis I disorders, research version, non-patient edition (SCID-I/NP). New York: Biometrics Research, New York State Psychiatric Institute.

Fitzgibbon, M. L., Prewitt, T. E., Blackman, L. R., Simon, P., Luke, A., Keys, L. C., \& Singh, V. (1998). Quantitative assessment of recruitment efforts for prevention trials in two diverse black populations. Preventive Medicine, 27, 838-845.

Fu, S. S., Kodl, M. M., Joseph, A. M., Hatsukami, D. K., Johnson, E. O., Breslau, N., \& Bierut, L. (2008). Racial/ethnic disparities in the use of nicotine replacement therapy and quit ratios in lifetime smokers ages 25 to 44 years. Cancer Epidemiology, Biomarkers, \& Prevention, 17, $1640-1647$.

Gandhi, K. K., Foulds, J., Steinberg, M. B., Lu, S. E., \& Williams, J. M. (2009). Lower quit rates among African American and Latino menthol cigarette smokers at a tobacco treatment clinic. International Journal of Clinical Practice, 63, 360-367.

Gariti, P., Levin, S., Whittingham, T., Barou, D., Kampman, K. M., Lynch, K., \& Alterman, A. (2008). Why do those who request smoking treat- ment fail to attend the first appointment? Journal of Substance Abuse Treatment, 35, 2-67.

Garrow, J. S., \& Webster, J. (1985). Quetelet's index (W/H2) as a measure of fatness. International Journal of Obesity, 9, 147-153.

Giovino, G. A., Sidney, S., Gfroerer, J. C., O’Malley, P. M., Allen, J. A., \& Richter, P. A. (2004). Epidemiology of menthol cigarette use. Nicotine \& Tobacco Research, 6, S67-S81.

Gonzales, D., Rennard, S. I., Nides, M., Oncken, C., Azoulay, S., Billing, C. B., \& Reeves, K. R. (2006). Varenicline, an $\alpha \mathrm{a} \beta 2$ nicotinic acetylcholine receptor partial agonist, vs. sustained-release bupropion and placebo for smoking cessation. Journal of General Internal Medicine, 296, 47-55.

Harris, K. J., Ahluwalia, J. S., Catley, D., Okuyemi, K. S., Mayo, M. S., \& Resnicow, K. (2003). Successful recruitment of minorities into clinical trials: The Kick It at Swope project. Nicotine \& Tobacco Research, 5, 575-584.

Heatherton, T. F., Kozlowshi, L. T., Frecker, R. C., \& Fagerström, K. (1991). The Fagerström Test for Nicotine Dependence: A revision of the Fagerström tolerance questionnaire. British Journal of Addiction, 86, 1119-1127.

Hjalmarson, A. I., Franzon, M., Westin, A., \& Wiklund, O. (1994). Effect of nicotine nasal spray on smoking cessation. A randomized, placebocontrolled, double-blind study. Archives of Internal Medicine, 154, 2567-2572.

Horner, M. J., Ries, L. A. G., Krapcho, M., Neyman, N., Aminou, R., Howlader, N., \& Edwards, B. K. (Eds.). (2009). SEER Cancer Statistics Review, 1975-2006, National Cancer Institute. Bethesda, MD. Retrieved from http://seer.cancer.gov/csr/1975_2006/

Hurt, R. D., Sachs, D. P., Gliver, E. D., Offorf, K. P., Johnston, J. A., Dale, L. C., \& Sullivan, P. M. (1997). A comparison of sustained-release bupropion and placebo for smoking cessation. New England Journal of Medicine, 337, 1195-1202.

Jeffries, S. K., Choi, W., Butler, J., Harris, K. J., \& Ahluwalia, J. S. (2005). Strategies for recruiting African-American residents of public housing developments into a randomized controlled trial. Ethnicity \& Disease, $15,773-778$.

Jorenby, D. E., Hays, J. T., Rigotti, N. A., Azoulay, S., Watsky, E. J., Williams, K. E., \& Reeves, K. R. (2006). Efficacy of varenicline, an $\alpha \mathrm{a} \beta 2$ nicotinic acetylcholine receptor partial agonist, vs placebo or sustained-release bupropion for smoking cessation: A randomized controlled trial. Journal of General Internal Medicine, 296, 56-63.

Jorenby, D. E., Leischow, S. J., Nides, M. A., Rennard, S. I., Johnston, J. A., Hughes, A. R., \& Baker, T. B. (1999). A controlled trial of sustained-release bupropion, a nicotine patch, or both for smoking cessation. New England Journal of Medicine, 340, 685-691.

Kaiser Family Foundation. (2001). Characteristics of persons living with AIDS at the end of 1999. Retrieved from statehealthfacts.org

Kiefe, C. I., Williams, O. D., Lewis, C. E., Allison, J. J., Sekar, P., \& Wagenknecht, L. E. (2001). Ten-year changes in smoking among young adults: Are racial differences explained by socioeconomic factors in the CARDIA study? American Journal of Public Health, 91, 213-218.

King, A. C., \& Cao, D. (2010, February). Naltrexone effects on smoking cessation quit rates, urges, and weight gain: An examination of sex differences. Paper presentation at the annual conference of the Society for Research on Nicotine and Tobacco, Baltimore.

King, A. C., Cao, D., Southard, C., \& Matthews, A. (2010, February). Racial disparities in screening and eligibility determination in a smoking cessation clinical trial. Paper presented at the annual conference of the Society for Research on Nicotine and Tobacco, Baltimore.

King, A. C., de Wit, H., Riley, R. C., Cao, D., Niaura, R., \& Hatsukami, D. (2006). Efficacy of naltrexone in smoking cessation: A preliminary study and an examination of sex differences. Nicotine \& Tobacco Research, 8, 671-682.

King, A. C., Sánchez-Johnsen, L., Van Orman, S., Cao, D., \& Matthews, 
A. K. (2008). A pilot community-based intensive smoking cessation intervention in African Americans: Feasibility, acceptability, and early outcome indicators. Journal of the National Medical Association, 100, 208-217.

King, G., Polednak, A., Bendel, R. B., Vilsaint, M. C., \& Nahata, S. B. (2004). Disparities in smoking cessation between African Americans and Whites: 1990-2000. American Journal of Public Health, 94, 19651971.

Kreuter, M. W., Lukwago, S. N., Bucholtz, R. D., Clark, E. M., \& Sanders-Thompson, V. (2003). Achieving cultural appropriateness in health promotion programs: Targeted and tailored approaches. Health Education \& Behavior, 30, 133-146.

Lacey, L. P., Manfredi, C., Balch, G., Warnecke, R. B., Allen, K., \& Edwards, C. (1993). Social support in smoking cessation among black women in Chicago public housing. Public Health Reports, 108, 387394.

Lee, R. E., McGinnis, K. A., Sallis, J. F., Castro, C. M., Chen, A. H., \& Hickmann, S. A. (1997). Active vs. passive methods of recruiting ethnic minority women to a health promotion program. Annals of Behavioral Medicine, 19, 378-384.

Lorence, D. P., Park, H., \& Fox, S. (2006). Racial disparities in health information access: Resilience of the digital divide. Journal of Medical Systems, 30, 241-249.

Matthews, A. K., Sánchez-Johnsen, L., \& King, A. C. (2009). Development of a culturally targeted smoking cessation intervention for African American smokers. Journal of Community Health, 34, 480-492.

Mazas, C. A., \& Wetter, D. W. (2003). Smoking cessation interventions among African Americans: Research needs. Cancer Control, $10(5$ Suppl), 87-89.

Nollen, N. S., Mayo, M. S., Sanderson Cox, L., Okuyemi, K. S., Kaur, H., \& Ahluwalia, J. S. (2006). Predictors of quitting among African American light smokers enrolled in a randomized, placebo-controlled trial. Journal of General Internal Medicine, 21, 590-595.

O’Malley, S. S., Cooney, J. L., Krishnan-Sarin, S., Dubin, J. A., McKee, S. A., Cooney, N. L., \& Jatlow, P. (2006). A controlled trial of naltrexone augmentation of nicotine replacement therapy for smoking cessation. Archives of Internal Medicine, 166, 667-674.

Pérez-Stable, E. J., Herrera, B., Jacob, P. III, \& Benowitz, N. L. (1998). Nicotine metabolism and intake in black and white smokers. Journal of General Internal Medicine, 280, 152-156.

Piper, M. E., Fox, B. J., Welsch, S. K., Fiore, M. C., \& Baker, T. B. (2001). Gender and racial/ethnic differences in tobacco-dependence treatment: A commentary and research recommendations Nicotine \& Tobacco Research, 3(4), 291-7.

Robles, G. I., Singh-Franco, D., \& Ghin, H. L. (2008). A review of the efficacy of smoking-cessation pharmacotherapies in nonwhite populations. Clinical Therapeutics, 30, 800-812.

Royce, J. M., Hymowitz, N., Corbett, K., Hartwell, T. D., \& Orlandi, M. A. (1993). Smoking cessation factors among African Americans and Whites. American Journal of Public Health, 83, 220-226.

Schneider, N. G., Olmstead, R., Nilsson, F., Mody, F. V., Franzon, M., \&
Doan, K. (1996). Efficacy of a nicotine inhaler in smoking cessation: A double-blind, placebo controlled trial. Addiction, 91, 1293-1306.

Shaya, F. T., Gbaravor, C. M., Huiwen, K. Y., Agyeman-Dauh, M., \& Saunders, E. (2007). A perspective on African American participation in clinical trials. Contemporary Clinical Trials, 28, 213-217.

Shiffman, S. (2009). Light and intermittent smokers: Background and perspective. Nicotine \& Tobacco Research, 11, 122-125.

Shiffman, S., Dresler, C. M., Hajek, P., Gilburt, S. J., Targett, D. A., \& Strahs, K. R. (2002a). Efficacy of a nicotine lozenge for smoking cessation. Archives of Internal Medicine, 162, 1267-1276.

Shiffman, S., Rolf, C. N., Hellebusch, S. J., Gorsline, J., Gorodetzky, C. W., Chiang, Y. K., \& Di Marino, M. E. (2002b). Real-world efficacy of prescription and over-the-counter nicotine replacement therapy. Addiction, 97, 505-516.

Signorello, L. B., Cai, Q., Tarone, R. E., McLaughlin, J. K., \& Blot, W. J. (2009). Racial differences in serum cotinine levels of smokers. Disease Markers, 27, 187-92.

Staffileno, B. A., \& Coke, L. A. (2006). Recruiting and retaining young, sedentary, hypertension-prone African American women in a physical activity intervention study. Journal of Cardiovascular Nursing, 21, $208-216$.

Targonski, P. V., Persky, V. W., Orris, P., \& Addington, W. (2005). Trends in asthma mortality among African Americans and Whites in Chicago, 1968 through 1991. American Journal of Public Health, 84, 1830-1833. Illinois Department of Health.

Tobacco Burden in Illinois. Retrieved from http://www.idph.state.il.us/ cancer/factsheets/burden.htm

Tonnesen, P., Norregaard, J., Mikkelsen, K., Jorgensen, S., \& Nilsson, F. (1993). A double-blind trial of a nicotine inhaler for smoking cessation. Journal of General Internal Medicine, 269, 1268-71.

Tonstad, S., Tonnesen, P., Hajk, P., Williams, K. E., Billing, C. B., \& Reeves, K. R. (2006). Effect of maintenance therapy with varenicline on smoking cessation: A randomized controlled trial. Journal of General Internal Medicine, 296, 64-71.

U.S. Census Bureau. (2000). Chicago Community Area (CCA) Profiles. Census Data for the City of Chicago.

Wagenknecht, L. E., Cutter, G. R,. Haley, N. J., Sidney, S., Manolio, T. A., Hughes, G. H., \& Jacobs, D. R. (1990). Racial differences in serum cotinine levels among smokers in the Coronary Artery Risk Development in (Young) Adults study. American Journal of Public Health, 80, $1053-6$.

Webb, M. S., Seigers, D., \& Wood, E. A. (2009). Recruiting African American smokers into intervention research: Relationships between recruitment strategies and participant characteristics. Research in Nursing \& Health, 32, 86-95.

Wong, G. Y., Wolter, T. D., Croghan, G. A., Croghan, I. T., Offord, K. P., \& Hurt, R. D. (1999). A randomized trial of naltrexone for smoking cessation. Addiction, 94, 1227-1237.

Woods, M. N., Harris, K. J., Mayo, M. S., Catley, D., Scheibmeir, M., \& Ahluwalia, J. S. (2002). Participation of African Americans in a smoking cessation trial: A quantitative and qualitative study. Journal of the National Medical Association, 94, 609-618. 